ADVANCES IN INTELLIGENT SYSTEMS AND COMPUTING 221

Javier Bajo Pérez et al. (Eds.)

\title{
Trends in Practical \\ Applications of Agents \\ and Multiagent Systems
}

11th International Conference on Practical Applications of Agents and Multi-Agent Systems

Springer 


\section{Advances in Intelligent Systems and Computing}

\section{Volume 221}

Series Editor

J. Kacprzyk, Warsaw, Poland 
Javier Bajo Pérez · Juan Manuel Corchado Rodríguez Johannes Fähndrich · Philippe Mathieu Andrew Campbell

María del Carmen Suárez-Figueroa

Emmanuel Adam · Antonio Fernández-Caballero

Ramon Hermoso · María N. Moreno

Editors

Trends in Practical

Applications of Agents

and Multiagent Systems

11th International Conference on Practical Applications of Agents and Multi-Agent Systems

Springer 


\section{Editors}

Javier Bajo Pérez

Departamento de Inteligencia Artificial

Facultad de Informática

Universidad Politécnica de Madrid

Madrid, España

Juan Manuel Corchado Rodríguez

Departamento de Informática y Automática

Facultad de Ciencias

Universidad de Salamanca

Salamanca, Spain

Johannes Fähndrich

Technische Universität Berlin

Faculty of Electrical Engineering and Computer Science

Berlin, Germany

Philippe Mathieu

Université des Sciences et Technologies de Lille

LIFL (Laboratoire d'Informatique Fondamentale de Lille)

Villeneuve d'Ascq Cedex, France

Andrew Campbell

Department of Computer Science

Dartmouth College

Hanover

USA

ISSN 2194-5357

ISBN 978-3-319-00562-1

DOI 10.1007/978-3-319-00563-8

ISSN 2194-5365 (electronic)

ISBN 978-3-319-00563-8 (eBook)

Springer Cham Heidelberg New York Dordrecht London

Library of Congress Control Number: 2013937540

(c) Springer International Publishing Switzerland 2013

This work is subject to copyright. All rights are reserved by the Publisher, whether the whole or part of the material is concerned, specifically the rights of translation, reprinting, reuse of illustrations, recitation, broadcasting, reproduction on microfilms or in any other physical way, and transmission or information storage and retrieval, electronic adaptation, computer software, or by similar or dissimilar methodology now known or hereafter developed. Exempted from this legal reservation are brief excerpts in connection with reviews or scholarly analysis or material supplied specifically for the purpose of being entered and executed on a computer system, for exclusive use by the purchaser of the work. Duplication of this publication or parts thereof is permitted only under the provisions of the Copyright Law of the Publisher's location, in its current version, and permission for use must always be obtained from Springer. Permissions for use may be obtained through RightsLink at the Copyright Clearance Center. Violations are liable to prosecution under the respective Copyright Law.

The use of general descriptive names, registered names, trademarks, service marks, etc. in this publication does not imply, even in the absence of a specific statement, that such names are exempt from the relevant protective laws and regulations and therefore free for general use.

While the advice and information in this book are believed to be true and accurate at the date of publication, neither the authors nor the editors nor the publisher can accept any legal responsibility for any errors or omissions that may be made. The publisher makes no warranty, express or implied, with respect to the material contained herein.

Printed on acid-free paper

Springer is part of Springer Science+Business Media (www.springer.com) 


\section{Contents}

\section{Special Session on Agents Behaviours and}

Artificial Markets (ABAM)

A Stylized Software Model to Explore the Free Market Equality/Efficiency Tradeoff

Hugues Bersini, Nicolas van Zeebroeck

Towards a Dynamic Negotiation Mechanism for QoS-Aware

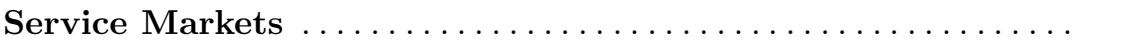

Claudia Di Napoli, Paolo Pisa, Silvia Rossi

\section{Special Session on Agents and Mobility (AM)}

D3S - A Distributed Storage Service

Rui Pedro Lopes, Pedro Sernadela

Evaluation of the Color-Based Image Segmentation

Capabilities of a Compact Mobile Robot Agent Based

on Google Android Smartphone ...................

Dani Martinez, Javier Moreno, Davinia Font, Marcel Tresanchez,

Tomàs Pallejà, Mercè Teixidó, Jordi Palacín

Distributed and Specialized Agent Communities

Jesús Ángel Román, Sara Rodríguez, Juan Manuel Corchado

A Gateway Protocol Based on FIPA-ACL for the New Agent Platform PANGEA

Alejandro Sánchez, Gabriel Villarrubia, Carolina Zato,

Sara Rodríguez, Pablo Chamoso

Applying Classifiers in Indoor Location System

Gabriel Villarubia, Francisco Rubio, Juan F. De Paz, Javier Bajo,

Carolina Zato 
Geo-localization System for People with Cognitive

Disabilities

João Ramos, Ricardo Anacleto, Paulo Novais, Lino Figueiredo, Ana Almeida, José Neves

\section{Special Session on Intelligent Components Producing and Consuming Knowledge and Data (ICP)}

Adding Sense to Patent Ontologies: A Representation

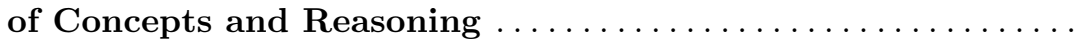

Maria Bermudez-Edo, Manuel Noguera, Nuria Hurtado-Torres, María Visitación Hurtado, José Luis Garrido

Representation of Clinical Practice Guideline Components in OWL

Tiago Oliveira, Paulo Novais, José Neves

\section{Special Sessions COoperative and RE-configurable} MultiAgent System (COREMAS)

Dynamically Maintaining Standards Using Incentives . . . . . . .

Ramón Hermoso, Henrique Lopes Cardoso

Self-organizing Prediction in Smart Grids through Delegate

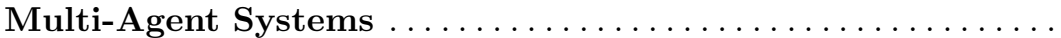
Leo Rutten, Paul Valckenaers

Holonic Recursiveness with Multi-Agent System Technologies .

Sonia Suárez, Paulo Leitao, Emmanuel Adam

Special Session on Multi-Agent Systems for Multi-sensor Activity Interpretation (MASMAI)

Intelligent Energy Management System for the Optimization of Power Consumption.

Vicente Botón-Fernández, Máximo Pérez Romero, Adolfo Lozano-Tello, Enrique Romero Cadaval

Find It - An Assistant Home Agent

Ângelo Costa, Ester Martinez-Martin, Angel P. del Pobil, Ricardo Simoes, Paulo Novais 
Efficient People Counting from Indoor Overhead Video

Camera .................................... 129

Juan Serrano-Cuerda, José Carlos Castillo, Marina V. Sokolova,

Antonio Fernández-Caballero

Modeling Intelligent Agents to Integrate a Patient

Monitoring System .

Gabriel Pontes, Carlos Filipe Portela,

Rui Rodrigues, Manuel Filipe Santos,

José Neves, António Abelha, José Machado

\section{Special Session on Self-Explaining Agents (SEA)}

Towards Self-Explaining Agents. . . . . . . . . . . . . . . . . . . 147

Johannes Fähndrich, Sebastian Ahrndt, Sahin Albayrak

Semi-automated Generation of Semantic Service

Descriptions ............................. 155

Nils Masuch, Philipp Brock, Sahin Albayrak

Patience in Group Decision-Making with Emotional

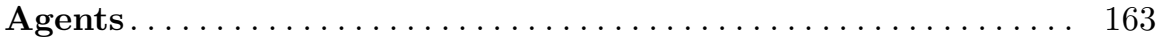

Denis Mušić

\section{Special Session on Web Mining and Recommender Systems (WebMiReS)}

Analysis of Web Usage Data for Clustering Based

Recommender System ......................... 171

Shafiq Alam, Gillian Dobbie, Patricia Riddle, Yun Sing Koh

Multi-label Classification for Recommender Systems . . . . . . . 181

Dolly Carrillo, Vivian F. López, María N. Moreno

If It's on Web It's Yours! . . . . . . . . . . . . . . . . . . . . . . . . . . 189

Abdul Mateen Rajput

User Assistance Tool for a WebService ERP . . . . . . . . . . . 193

Israel Carlos Rebollo Ruiz, Manuel Graña Romay

TV-SeriesRec: A Recommender System Based on Fuzzy

Associative Classification and Semantic Information . . . . . . . 201

Diego Sánchez-Moreno, Ana Belén Gil, María N. Moreno

Author Index . . . . . . . . . . . . . . . . . . . . . . . . . . . 209 


\title{
Applying Classifiers in Indoor Location System
}

\author{
Gabriel Villarubia, Francisco Rubio, Juan F. De Paz, \\ Javier Bajo, and Carolina Zato
}

\begin{abstract}
Research in indoor location has acquired a growing importance during the recent years. The main objective is to obtain functional systems able of providing the most precise location, identification and guidance in real time. Currently, none of the existing indoor solutions have obtained location or navigation results as precise as the ones provided by the analog systems used outdoor, such as GPS. This paper presents an indoor location system based on Wi-Fi technology which, from the use of intensity maps and classifiers, allows effective and precise indoor location.
\end{abstract}

Keywords: indoor location system, classifiers, Wi-Fi.

\section{Introduction}

Nowadays, indoor location is one of the most promising research fields in mobile computing [7], and it is possible to find several studies. To obtain precise information about the location of objects or people inside building can provide very useful information to develop services. Some of the most relevant of these services are those oriented to identification-based access control, location-based security, location-aware computing, etc.. These services are deployed in indoor environments, such as hospitals, factories, shopping malls, or even as complementary systems to GPS (Global Positioning System). The main challenge in current indoor location systems is to obtain precise measures with a reasonable cost in infrastructure.

Gabriel Villarubia · Francisco Rubio · Juan F. De Paz · Carolina Zato

Department of Computer Science and Automation, University of Salamanca,

Plaza de la Merced, s/n, 37008, Salamanca, Spain

e-mail: $\{g v g$, paco_rg, fcofds, carol_zato\}@usal.es

Javier Bajo

Departamento de Inteligencia Artificial, Facultad de Informática,

Universidad Politécnica de Madrid, Madrid, Spain

e-mail: jbajo@fi.upm.es

J.B. Pérez et al. (Eds.): Trends in Prac. Appl. of Agents \& Multiagent Syst., AISC 221, pp. 53-58.

DOI: 10.1007/978-3-319-00563-8_7 $\quad$ C Springer International Publishing Switzerland 2013 
The main reason to explain why it is not possible to find effective indoor solutions is due to technical and financial reasons. If we take into consideration a GPS system, we simply need a physical device that connects to a finite number of satellites in open spaces. However, in an open space, it is required to make use of a technological infrastructure with a considerable number of fixed devices that are used as beacons or readers, and notably improve the cost of the system. Amongst the technologies that are currently used most in the development of Real-Time Location Systems are, RFID (Radio Frequency IDentification) [8] [10], Wi-Fi y ZigBee [8] [9]. Nowadays, some of the existing indoor location engines make use of 802.11 technology also known as $\mathrm{Wi}-\mathrm{Fi}$, and have acquired a growing importance due to the reduced and reasonable cost of these systems compared to the alternatives. Moreover, Wi-Fi provides a good number of advantages: easy deployment and is integrated in most of the current electronic devices, wide range of existing networks installed in several buildings and locations.

This paper presents a study aimed at obtaining the design of an innovative Wi$\mathrm{Fi}$ indoor location engine, able of providing location-aware Information of people or objects inside a building. The use of Wi-Fi technology for location systems is mainly based on intensity maps constructed from RSSI levels in different zones. The maps are used as a basis to obtain classifications. The classifiers use the data of the maps and the data obtained from the devices to determine the position of a person inside a building. The system presented in this paper has been adapted to be installed in resource-constrained devices. In this way it is not necessary to connect to databases to obtain information for the classification, which notable improve the performance of the system and the fault tolerance.

This article is divided as follows: section 2 describes the state of the art; section 3 presents the proposed model; section four describes the results obtained and the conclusions respectively.

\section{Background}

There exist three main algorithms that are used by real-time location systems to determine the location of the mobile nodes (tags): Triangulation, Fingerprinting and Multilateration [4]. Triangulation allows obtaining location coordinates by means of the calculus of the length of the sides of the triangle from the angles of the received signal in each of the antennas, which requires at least 3 reference points. Fingerprinting, also known as signpost or symbolic location, is based on the study of the characteristics of the each of the location zones, obtaining measurements of the radiofrequency characteristics and estimating the influence area of each tag. [5]. Multilateration estimates the distances between readers and tags, taking into account parameters as RSSI (Received Signal Strength Indication) or TDOA (Time Difference Of Arrival) [6], in such way that the intersection of the estimated distances from each tag to three or more fixed nodes determines the point where the tags are identified. Multilateration provides better results that triangulation when it is used outdoors, but its performance is notably reduced 
indoors. The reason is fluctuation of the RSSI levels in indoor environments due to the presence of different elements (people, objects, animals). Besides, multilateration is based on the estimation of distances, and it is necessary a previous estimation of the RSSI values, which is difficult because the RSSI values change constantly.

Location techniques based on triangulation and multilateration cannot be considered as efficient because the signals suffer several attenuations caused by the elements present in the rooms. In these cases, it is recommended to use heuristics for the classification process and perform training to obtain location algorithms that improve the precision. The heuristics allow the collection of possible measurements in specific positions and to use these measurements to calculate the most probable position.

\section{Proposed Reasoning System}

Classification techniques facilitate the association of cases to the existing groups. The behavior of these algorithms is similar to the algorithms used for clustering, but they are too much simple in most of the cases. The existing techniques can be grouped in the following categories depending on the nature of the algorithms:

- Decision rules and decision trees.

- Probabilistic models: Naive Bayes [11], Bayesian networks [1] [2] [3].

- Fuzzy logic: K-NN (K-Nearest Neighbours), NN (K-Nearest Neighbours).

- Function search: Sequential Minimal Optimization (SMO) [12].

- Artificial neural networks.

Specifically, the proposed system has integrated a bayesian network to estimate the probabilities of belonging to the points previously scanned in the intensity maps. The intensity maps obtained indoors are created using the parameters shown in Table 1. Each of the rows in Table 1 contains the information of all the Wi-Fi networks scanned in that moment and identified by a coordinate $(\mathrm{x}, \mathrm{y})$. The rows can contain more or less columns depending on the scanned intensities.

Table 1 Format of the measurements in the intensity maps

\begin{tabular}{lllllll}
\hline$x$ & $y$ & SSID & BSSID & RSSI & SSID & $\ldots$ \\
\hline
\end{tabular}

The scanned measurements, represented using the format shown in Table 1, are used to obtain the distances that are used by the bayesian network which is trained and configured for further estimations. In order to build Bayesian networks, it is first necessary to establish search mechanisms that can generate the DAG (Directed Acyclic Graph) using a set of heuristics that can reduce the number of combinations and generate the final Bayesian network. There are various Bayesian network search mechanisms, including tabu search [1], conditional independence 
[2], K2 [1], HillClimber [1], TAN (Tree Argumented Naive Bayes) [3]. In this work, we have used conditional independence. This algorithm is based on the calculation of the conditional Independence test for the variables to generate a DAG that can obtain the probability estimates. If the variables being studied are independent, it will not be possible to generate a Bayesian network with good results, but in this work this is not a problem with the independence of the variables. The attribute for the classification in the bayesian network is defined as the union of $\mathrm{x}$ and $\mathrm{y}$.

\section{$4 \quad$ Results and Conclusions}

To analyze the overall performance of the system, we defined a case study in the University of Salamanca. The first step was to calibrate the map for the first floor of the Physics building at the Faculty of Sciences. During the calibration process, we obtained different signal measurements in the corridors of the floor. The classifier was constructed from these data. The total number of measurements was 380, as shown in Figure 1a. The area of the floor of the building was approximately $1700 \mathrm{~m} 2$. The floor was equipped with 4 fixed access points spread across the floor of the building. Moreover, some signals emitted by access points that went out and lit corresponding to offices and laboratories were detected. The access points were the infrastructure nodes available in the university, and it was not necessary to introduce any additional access points to reduce the system error. Figure shows a screenshot obtained from the mobile device used for the experiment.
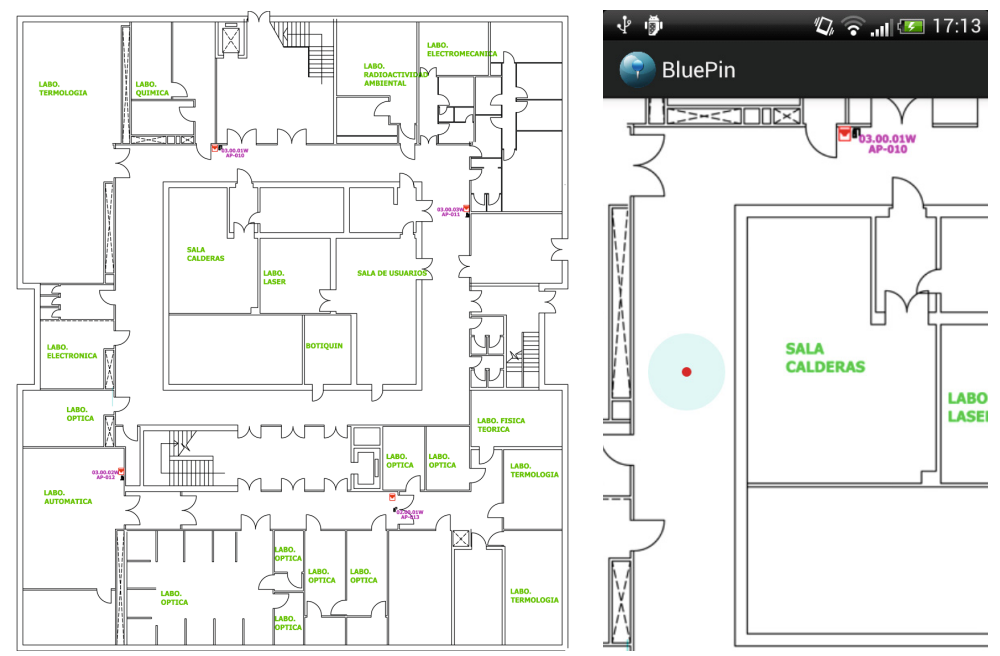

Fig. 1 A) First floor of the Physics building. B) Mobile interface of the system. 
The system was evaluated obtaining measures in different points and estimating the position. We obtained 332 new measurements and estimated the corresponding positions. The results obtained are shown in Table 2. The absolute mean error obtained is shown in the first column and, as can be seen in Table 2, the bayesian network provides the best value for the error, 2,4 meters. The average time to estimate the position is 0,65 milliseconds, which is good enough to be used in a mobile device. It is necessary that the average time has been obtained in a laptop. It has not been possible to measure the time in a mobile device, but the system perfectly worked in the mobile during the experiment.

Table 2 Errors and time to estimate positions using different classifiers

\begin{tabular}{lll}
\hline Error meters & Time millisecond & Classifier \\
\hline 2,414 & 0,652632 & .BayesNet \\
3,040 & 2,126316 & NaiveBayes \\
3,040 & 1,957895 & NaiveBayesUpdateable \\
4,147 & 37,989475 & SimpleLogistic \\
8,890 & 22,660526 & SMO \\
3,945 & 0,281579 & Ibk \\
8,299 & 11,03421 & LWL \\
2,857 & 14,047368 & KStar \\
9,430 & 0,078947 & AdaBoostM1 \\
3,830 & 0,281579 & AttributeSelectedClassifier \\
5,035 & 1,452632 & Bagging \\
4,256 & 6,481579 & ClassificationViaRegression \\
12,605 & 0,042105 & CVParameterSelection \\
4,325 & 0,2 & FilteredClassifier \\
4,306 & 8,652632 & LogitBoost \\
12,624 & 7,476316 & MultiClassClassifier \\
12,605 & 0,052632 & MultiScheme \\
3,826 & 0,4 & RandomCommittee \\
3,560 & 0,768421 & RandomSubSpace \\
12,605 & 0,047368 & Stacking \\
12,510 & 5,247368 & DecisionTable \\
4,572 & 1,760526 & JRip \\
13,370 & 0,044737 & OneR \\
6,488 & 1,639474 & PART \\
12,605 & 0,039474 & ZeroR \\
9,430 & 0,068421 & DecisionStump \\
7,819 & 0,255263 & J48 \\
14,142 & 90,007896 & LMT \\
3,346 & 0,415789 & RandomForest \\
2,918 & 0,092105 & RandomTree \\
2,649 & 0,194737 & REPTree \\
\hline & & \\
\hline & & \\
\hline
\end{tabular}


As a conclusion, it is possible to say that the work presented in this paper facilitates a new technique to locate objects and people using Wi-Fi signals in indoor environment, making use of the existing infrastructure networks. The system does not require the installation of additional access points or any other hardware to improve the system performance. Thus, the implementation cost is notably reduced compared to most of the existing systems. Our future work focuses on the incorporation of new measurements into the algorithm, such as GSM or 3G that can be combined with the Wi-Fi signals and improve the overall performance of the system.

Acknowledgments. This work has been supported by the Ministry of Economy and Competitiveness (INNPACTO) IPT-2011-0726-430000.

\section{References}

1. Bouckaert, R.R.: Bayesian Belief Networks: from Construction to Inference, Utrecht, Netherlands (1995)

2. Verma, T., Pearl, J.: An algorithm for deciding if a set of observed independencies has a causal explanation. In: Proc. of the Eighth Conference on Uncertainty in Artificial Intelligence, pp. 323-330 (1992)

3. Friedman, N., Geiger, D., Goldszmidt, M.: Bayesian Network Classifiers. Machine Learning 29, 131-163 (1997)

4. Glassner, A.: Principles of digital image synthesis. Morgan Kaufmann (1995)

5. Georgé, J.P., Gleizes, M.P., Glize, P.: Emergence of organisations, emergence of functions. In: Symposium on Adaptive Agents and Multi-Agent Systems, pp. 103-108 (2003)

6. De Paz, J.F., Rodríguez, S., Bajo, J., Corchado, J.M.: Multi-agent system for security control on industrial environments. International Transactions on System Science and Applications Journal 4(3), 222-226 (2008)

7. Chen, Y.-C., Chiang, J.-R., Chu, H.-H., Huang, P., Tsuid, A.W.: Sensor-Assisted WiFi Indoor Location System for Adapting to Environmental Dynamics (2011)

8. Razavi, R.S., Perrot, J.-F., Guelfi, N.: Adaptive modeling: An approach and a method for implementing adaptive agents. In: Ishida, T., Gasser, L., Nakashima, H. (eds.) MMAS 2005. LNCS (LNAI), vol. 3446, pp. 136-148. Springer, Heidelberg (2005)

9. Giunchiglia, F., Mylopoulos, J., Perini, A.: The tropos software development methodology: Processes, models and diagrams. In: AAMAS 2002 Workshop on Agent Oriented Software Engineering (AOSE 2002), pp. 63-74 (2002)

10. Tapia, D.I., De Paz, J.F., Rodríguez, S., Bajo, J., Corchado, J.M.: Multi-Agent System for Security Control on Industrial Environments. International Transactions on System Science and Applications Journal 4(3), 222-226 (2008)

11. Duda, R.O., Hart, P.: Pattern classification and Scene Analysis. John Wisley \& Sons, New York (1973)

12. John, C.: Platt Fast training of support vector machines using sequential minimal optimization. In: Advances in Kernel Methods, pp. 185-208 (1999) 